\title{
3D Analysis of a Fatigue Crack in Cast Iron Using Digital Volume Correlation of X-ray Tomographic Images
}

\author{
Joël Lachambre, Arnaud Weck, Julien Réthoré, Jean-Yves Buffière, and Jérôme Adrien
}

\begin{abstract}
Three-dimensional (3D) images of a thumbnail corner fatigue crack are obtained in a sample of nodular graphite cast iron using laboratory X-ray computed tomography. The crack is initiated in situ from an artificial defect created by laser machining, its development is followed in situ and Digital Volume Correlation (DVC) analysis of the 3D images gives access to the 3D displacement field at the tip of the crack (mainly mode I opening).
\end{abstract}

Keywords X-ray tomography $\bullet$ Digital volume correlation $\bullet$ 3D fatigue crack $\bullet$ In situ

\subsection{Introduction}

In their early stage of propagation, fatigue cracks have a strong three dimensional character. This is because, in structural materials, this phenomenon occurs at heterogeneities such as holes, surface scratches, harder or softer second phase particles, well oriented grain etc. Furthermore, once initiated, small cracks strongly interact with the surrounding grains and, at low stress levels, a large number of fatigue cycles is necessary before the propagation rate of a crack at the specimen surface is comparable to the propagation rate in the bulk of the specimen. Fatigue models tend to ignore this three dimensional aspect of crack growth partly because reliable experimental data was (and still is) lacking. This is a crucial issue because a very large fraction of the fatigue life of real components correspond to the growth of such small 3D cracks [1].

In this paper, a fatigue crack initiating from an artificial defects is characterised with X-ray micro-tomography and DVC. In previous studies, Limodin et al. [2] had used those two techniques to study crack opening/closure in a nodular cast iron. However the cracks had been obtained from larger samples and the influence of sample preparation (relaxation of stress) could not be excluded. In this work a method has been developed to initiate and grow a crack in a single sample thus providing a cleaver picture of the displacement field at the tip of a growing fatigue crack.

J. Lachambre $(\bowtie) \cdot J .-Y$. Buffière • J. Adrien

Laboratoire Materiaux, Ingenierie et Sciences (MATEIS), INSA-Lyon/CNRS-UMR 5510, 20 Av. Albert Einstein,

F-69621Villeurbanne, France

e-mail: joel.lachambre@insa-lyon.fr

A. Weck

University of Ottawa, 161 Louis Pasteur, Colonel By Hall - Room A205B, Ottawa, ON K1N 6N5, Canada,

J. Réthoré

Laboratoire de Mecanique des Contacts et des Structures (LaMCoS), INSA-Lyon/CNRS-UMR 5259,

20 Av. Albert Einstein, F-69621Villeurbanne, France 


\subsection{Experimental Methods}

\subsubsection{Samples}

The material used in this study is a nodular graphite cast iron (3.4 wt $\% \mathrm{C}, 2.6 \mathrm{wt} \% \mathrm{Si}, 0.05 \mathrm{wt} \% \mathrm{Mg}, 0.19 \mathrm{wt} \% \mathrm{Mn}$, $0.005 \mathrm{wt} \% \mathrm{~S}$, and $0.01 \mathrm{wt} \% \mathrm{P}$ ) with a homogeneous dispersion of graphite nodules which are used as natural markers for DVC [2]. Casting and appropriate heat treatments resulted in a ferritic matrix microstructure with a 14\% volume fraction of graphite nodules with an average diameter of $45 \mu \mathrm{m}$. Young's modulus, yield stress and Poisson's ratio are equal to $175 \mathrm{GPa}, 300 \mathrm{MPa}$, and 0.27 , respectively.

The solidification process of the nodular graphite cast iron can sometimes create large porosities $(\sim 300 \mu \mathrm{m})$; those are relatively rare and quite hard to detect in radiography. Ideally to act as a crack initiation site such a defect should be located in the middle of a $1.6 \times 1.6 \mathrm{~mm}^{2}$ cross-section, and therefore the cutting of the sample proved extremely difficult. Therefore an alternative solution is adopted: the initiation from well controlled artificial defect created by laser machining [3].

The crack is machined using a $1 \mathrm{kHz}$ repetition rate regeneratively amplified Ti:Sapphire laser system employing chirped pulse amplification. A pulse length (full-width half-maximum) of approximately $40 \mathrm{fs}$ is measured using an auto-correlator. A femtosecond laser is chosen in this work as it results in a negligible heat affected zone around the crack due to the high intensity of the laser pulse which directly vaporizes the material. For all experiments presented here, we used a rotating polarization obtained with a spinning zero order half wave plate. The beam is focused with a $0.25 \mathrm{~N}$.A. 10x microscope objective using an energy per pulse of $50 \mu \mathrm{J}$. Cast iron samples are placed on a computer controlled x-y translation stage for precise sample positioning under the laser beam. The crack is produced by translating the stage back and forth under the laser beam until the proper crack depth is obtained.

Two kind of defects corresponding to a corner notch and a surface notch have been studied. The notches have approximately a square shape $180 \times 180 \mu \mathrm{m}^{2}$ see Fig. 28.1) with an opening of $15 \mu \mathrm{m}$ and a tip radius (both measured at the sample surface) of about $7 \mu \mathrm{m}$.

\subsubsection{In Situ Cycling}

The cycling of the sample is performed in situ using a specially designed fatigue machine. In order to decrease the voxel size in the reconstructed 3D images, the sample must be as close as possible to the X-ray source, thus the sample is put into a long slender aluminium tube allowing the mechanical loading device and the load sensor to pass respectively under and above the
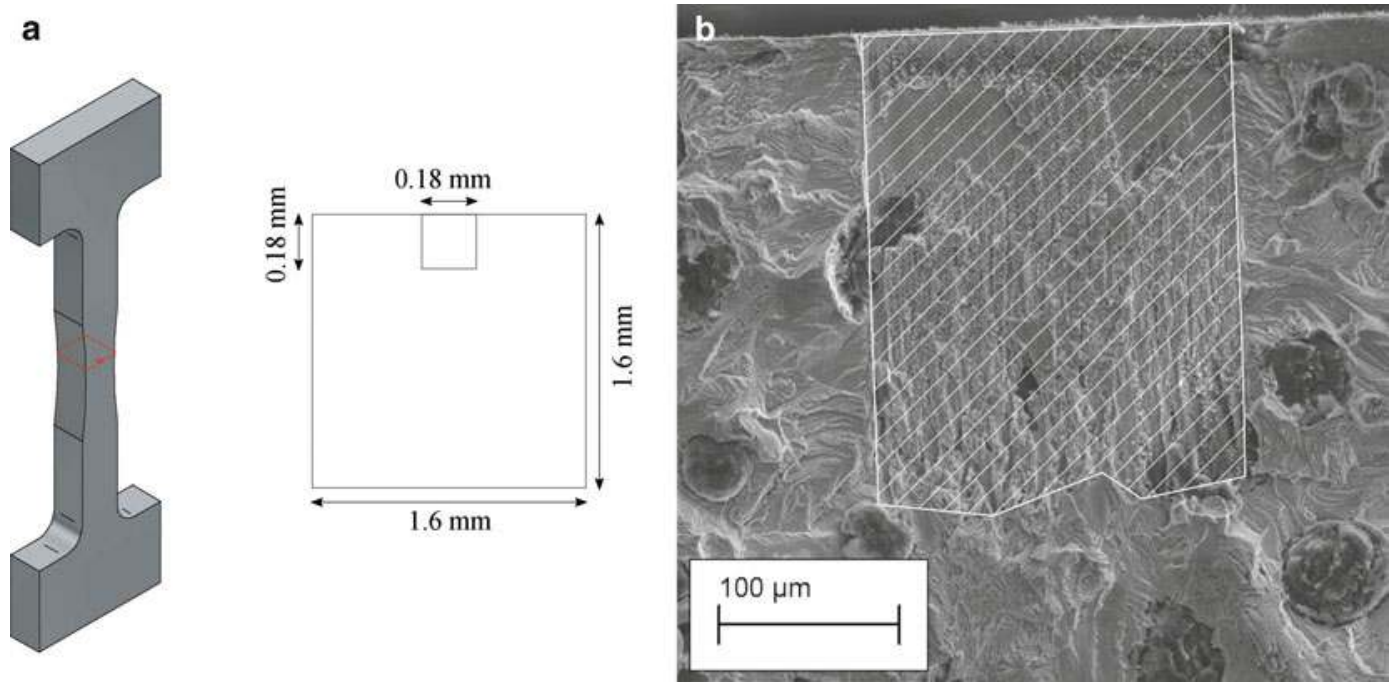

Fig. 28.1 (a) Schematic drawing of the fatigue sample showing the dog bone shape of the sample used and the location of the notch introduced in the square cross-section (b) SEM observation of the fracture surface showing the approximately square shape of the artificial defect introduced by laser machining 
a

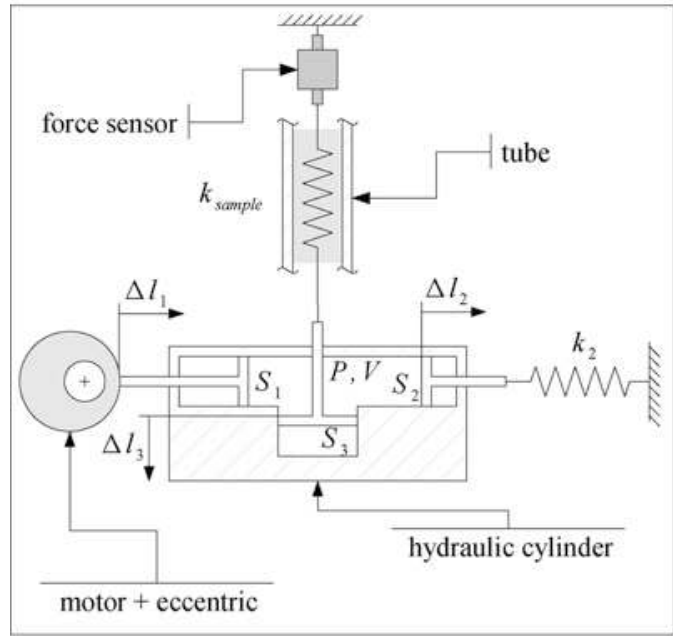

the applied load which is measured by the force sensor depend on the stiffness and the eccentric

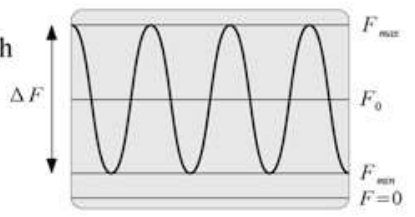

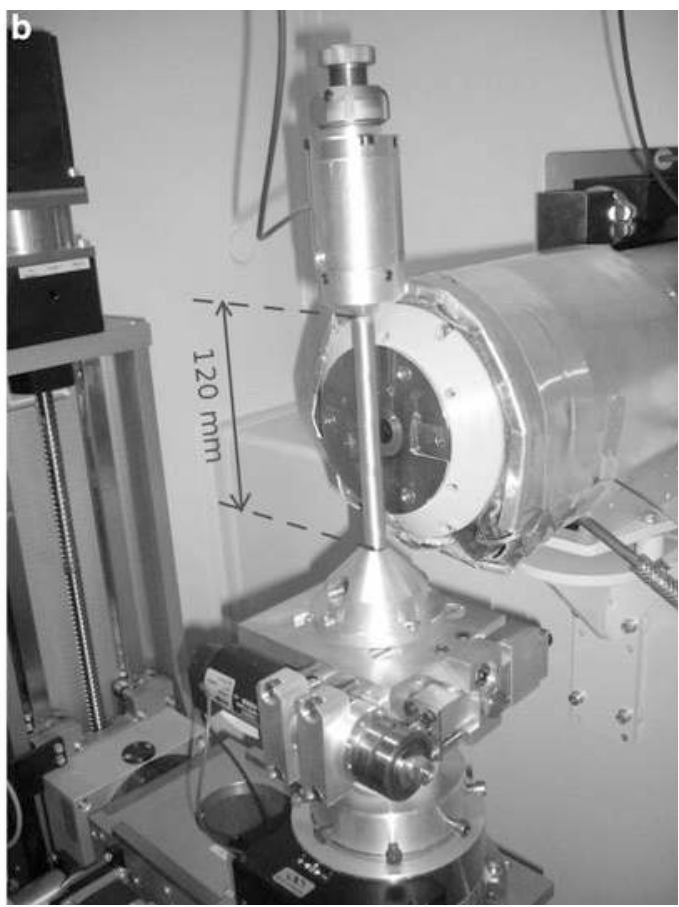

Fig. 28.2 (a) Schematic drawing showing the principle of cycling loading which uses hydraulic transmission (see the text for details), (b) Photograph of the machine installed in the tomograph (yellow part $=\mathrm{X}$-ray tube)

X-ray tube (see Fig. 28.2b). The tube diameter an thickness have to be kept as small as possible to be transparent to X-rays. In this work the diameter of the tube ( $\mathrm{Al} 2024$ alloy) is $16 \mathrm{~mm}$ and the thickness $1 \mathrm{~mm}$ [4].

High frequency cyclic loading is obtained via a combination of eccentric rotating cam and hydraulic transmission (see Fig. 28.2a). The eccentric is mounted on a position controlled motor pushing a cylinder (surface $S_{1}$ on Fig. 28.2a) which has a sinusoidal displacement $\Delta l_{1}$. The compression force is converted into a tensile force by a pull cylinder (surface $S_{3}$ ) which gives the displacement $\Delta l_{3}$. The choice of using an hydraulic cylinder enables to use a motor with high rotating speed $(1,800 \mathrm{rpm})$ and with relatively low torque $(0.58 \mathrm{Nm})$, the ratio $\frac{S_{1}}{S_{3}}$ gives the load magnification factor of the system (26 in this case). The mean load $F_{0}$ and its amplitude $\Delta F$ can be changed by adjusting of the stiffness of a leak cylinder (stiffness $k_{2}$ on Fig. 28.2a).

The motor is driven by a digital positioning controller connected to a computer using home-made software. The load sensor is also connected to computer using an analog-to-digital converter card.

The machine is designed to apply a uni-axial load to the sample (mode I opening of the crack). A test has been done to check this point: a smooth unnotched sample has been set in tension under two different loads (100 and $600 \mathrm{~N})$ and the corresponding displacement field has been analysed. Results of this analysis are shown in Fig. 28.3.

The DVC on this test has been done with 32 voxel size elements; as shown in Ref. [2], the uncertainty (corresponding to the standard deviation on a displacement field between two scans of an sample which is just moved of a few voxels between them) is about 0.05 voxels what explains the fluctuations in the displacement fields shown by Fig. 28.3. Mean strains can also be extracted from the displacement. A $0.1 \%$ strain is obtained in the loading direction while about one third of this value is estimated for the transverse directions. Further, shear strains are at least ten times smaller. This rough analysis confirms that the loading machine allows to performed quasi-uniaxial tension test.

\subsubsection{X-ray Tomography}

The standard laboratory tomograph available at MATEIS is manufactured by the Phoenix X-ray company. It is built inside a lead self-protected cabin which rather large dimensions (approximately $1.2 \times 1.5 \times 2 \mathrm{~m}^{3}$ ) that allows to install in situ devices. 


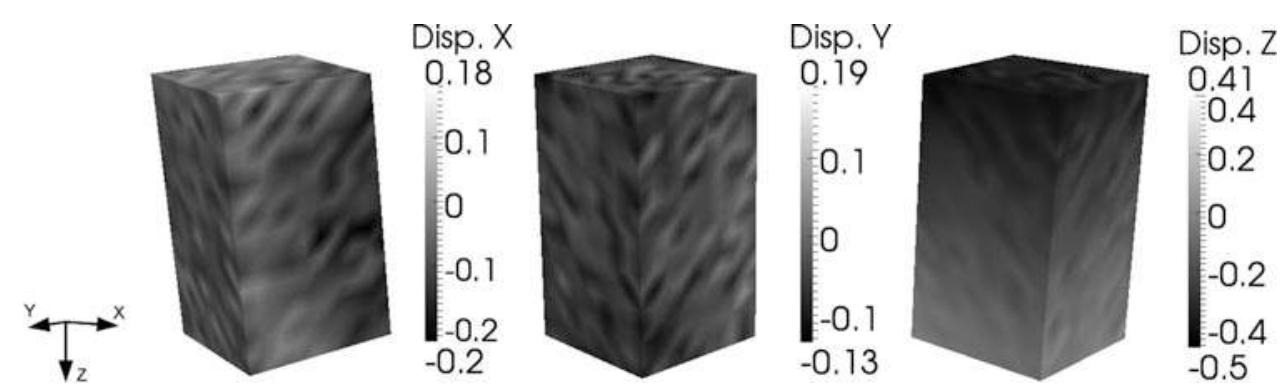

Fig. 28.3 Displacement field measured in a smooth tensile sample. The applied stress is along the Z. direction. The scale is given in voxel (1 voxel $=3.5 \mu \mathrm{m})$

The X-ray source is an open transmission nanofocus $\mathrm{X}$ ray tube which has been operated at an accelerating voltage of $85 \mathrm{kV}$. A tungsten target has been used. The tube delivers a cone $\mathrm{X}$ ray beam with a focus size that can be tuned between 1 and $6 \mu \mathrm{m}$. When the focus size is reduced, the geometrical blurring effect is reduced and, therefore, the image resolution increases. A reduction in the focus size is however accompanied by a reduction in the X-ray flux so that a trade off has to be reached between exposure time and resolution. In our case the minimum source/detector distance is imposed by the $\mathrm{Al}$ tube and set to $15 \mathrm{~mm}$ resulting in a voxel size of $3.5 \mu \mathrm{m}$.

The detector is a Paxscan ${ }^{\mathrm{TM}}$ amorphous silicon flat panel composed of 1,920 rows and 1,536 lines of square sensitive pixels (Physical size: $127 \times 127 \mu^{2}$ ).

A current of $174 \mu \mathrm{A}$ and a mean of three radiography with an exposure time of $1 \mathrm{~s}$ have been used. Seven hundred and twenty angular positions have been taken which corresponded to a scan time of $45 \mathrm{~min}$. The reconstruction time (weighted filtered back projection, Feldkamp algorithm) on a Desktop computer with a bi Intel xeon quad core processor, $3 \mathrm{GHz} \mathrm{CPU}$, 64 GBytes RAM, takes approximately $15 \mathrm{~min}$ for a $560 \times 560 \times 900$ voxels $3 \mathrm{D}$ image.

\subsubsection{In Situ Propagation}

The contrast of the crack on the radiographs is not large enough to quantify crack propagation. Therefore the only way to know how the crack grows in the interior of the sample is to record a tomographic scan. The crack shape evolution is studied through the residual of the DVC which correspond to the difference between the reference and the deformed and corrected volumes [2].

Once the sample is set up in the tomograph, two scans are recorded at low stress (40 MPa) and a scan at high stress (290 MPa). The sample is cycled in situ at constant a stress amplitude of $300 \mathrm{MPa}$ (load ratio: 0.1). In the beginning of the experiment 100,000 cycles are applied to the sample before a first scan is recorded. Then two scans are recorded every 15 kcycles, one at $40 \mathrm{MPa}$ and an other at $290 \mathrm{MPa}$, to monitor the crack evolution as described in [5]. The aim is to obtain from the residual map a "picture" of the crack. Because the cycling is performed at constant stress amplitude, the crack accelerates during propagation. Thus near the end of experiment smaller steps are used between two scans according to the approximate crack speed $\frac{d a}{d N}$ we observe.

Each specimen are cycled until fracture. In some cases, in addition to the two steps at 40 and $290 \mathrm{MPa}$, two other scans are recorded at intermediate loads in order to see the opening process.

\subsubsection{Digital Volume Correlation}

A C8-DVC multiscale algorithm with an eight node cubic element is used to compute the displacement fields in the loaded specimen. Details of the method can be found in Ref. [6]. Two kinds of correlation are performed during the experiment: first between a reference volume acquired before cycling under low stress and a deformed volume under high stress at each step 
of cycling. Then a correlation between the volume at low stress and the volume at high stress is also performed at each step. The first kind of analysis gives access to the crack shape evolution while the second is used to map the crack opening displacement (COD).

\subsection{Results}

\subsubsection{Crack Morphology}

The DVC analysis during cycling is carried out with 32 voxels elements, over a volume of $11 \times 11 \times 15 \mathrm{C} 8$ elements. This mesh is quite coarse considering that the corner crack at $180 \mathrm{kc}$ (see Fig. 28.4a) is contained in only eight elements. Nevertheless the crack morphology can be prickles estimated from the DVC residual.

Figure 28.4 illustrate the propagation of the crack of both kind of crack (corner and surface) based on the DVC residual. It can be seen that the crack shape is rapidly independent of the initial defect shape for both type of notches.

\subsubsection{Displacement Field}

An extended version of DVC called X-DVC (based on the principle of the extended finite element method. Ref. [7]) is used to post process the 3D images (between the scans at low and high stress).

The results of X-DVC on the corner and the surface crack are given in Fig. 28.5. As the position of the crack front is not known, a discontinuous enrichment is added over the whole cross-section.

The enrichment in the X-DVC gives access to the Crack Opening Displacement (COD). Figure 28.6 shows the evolution of the COD for five steps of cycling for the corner crack sample; it can be seen, as expected, that the COD is gradually increasing when the crack propagates.

Those displacement fields can be used to compute stress intensity factor values along the crack front using Williams series [8] as described in [2] in order to test existing models of crack propagation (e.g. the classical Paris law) wich are generally tested on $2 \mathrm{D}$, through crack. This work is under progress.

a
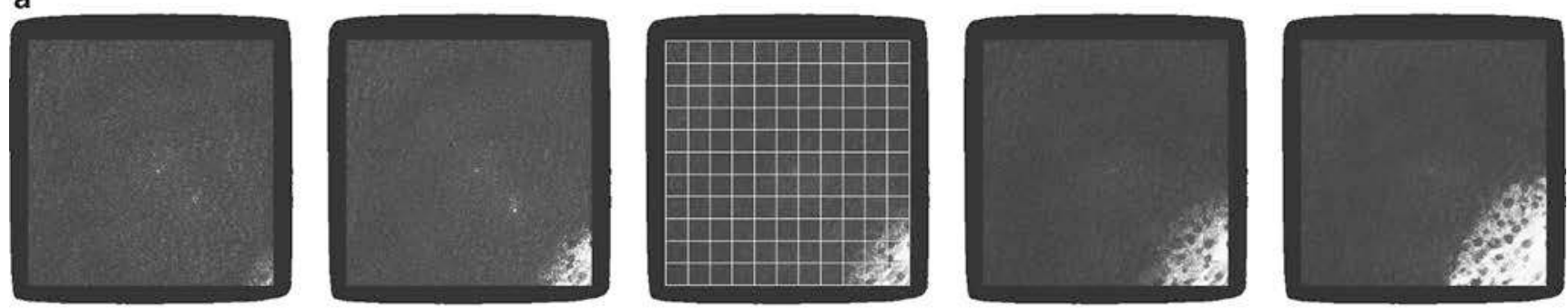

b
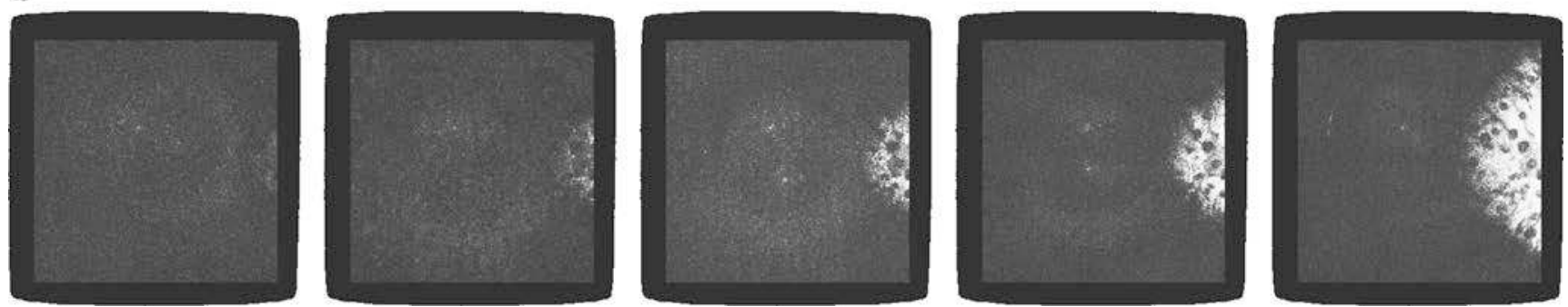

Fig. 28.4 Evolution of the DVC residual during the cycling. The white grid drawn over the corner crack at $180 \mathrm{kc}$ represent the elements used by the DVC analysis (the grey-scale is arbitrary). (a) corner crack evolution at $115 \mathrm{kc}, 155 \mathrm{kc}, 180 \mathrm{kc}, 190 \mathrm{kc}$ and $195 \mathrm{kc}$ (b) surface crack evolution at $160 \mathrm{kc}, 175 \mathrm{kc}, 190 \mathrm{kc}, 205 \mathrm{kc}$ and $240 \mathrm{kc}$ 


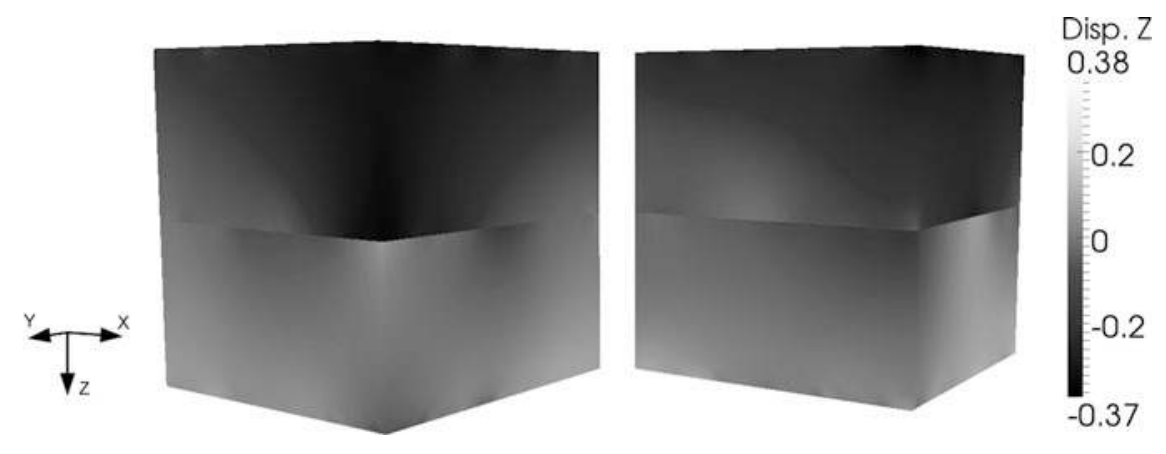

Fig. 28.5 Mode I component of the displacement field of the corner crack after $180 \mathrm{kc}(l e f t)$ and the surface crack after $200 \mathrm{kc}($ right). Those fields are results of X-DVC analysis. The scale is given in voxel $(1$ voxel $=3.5 \mu \mathrm{m})$
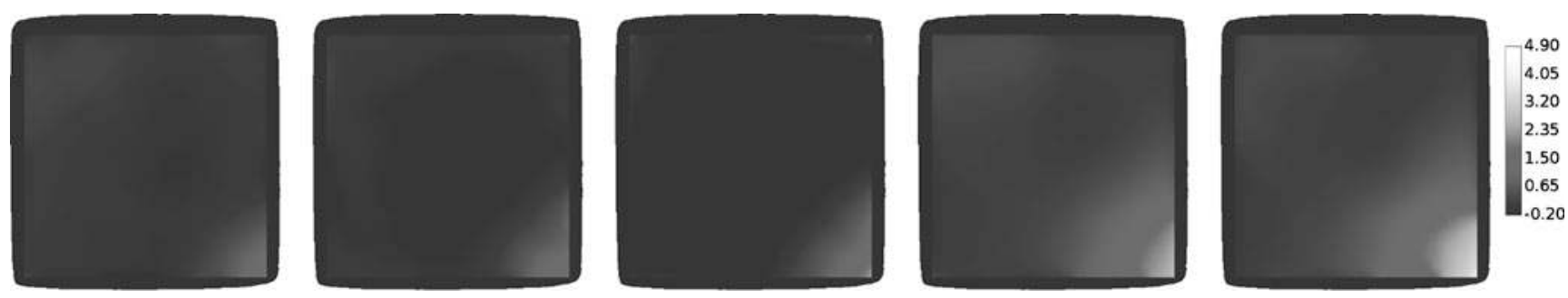

Fig. 28.6 Crack opening displacement evolution for the corner crack at $165 \mathrm{kc}, 180 \mathrm{kc}, 190 \mathrm{kc}, 195 \mathrm{kc}$ and $200 \mathrm{kc}$ (scale in $\mu \mathrm{m}$ )

\subsection{Conclusions}

The 3D propagation of a fatigue crack initiating from an artificial defect in a cast iron sample has been characterized in situ and analyzed using laboratory X-ray computed tomography and DVC.

The process of creating small artificial defects with laser beam gives an appropriate way to initiate a crack in this material. The crack propagation seems to be rapidly independent of the initial defect shape, after $150 \mathrm{kc}$ for the corner crack and $175 \mathrm{kc}$ for surface crack, in those test conditions, we don't see any influence of the notch shape.

The DVC analysis with a relatively coarse mesh provides during the cycling a valuable information on the propagation of the crack which allows to control the number of cycles to perform between each step.

The X-DVC analysis allows to estimate directly the crack opening displacement over the entire crack surface.

Acknowledgements This work was funded by the French ANR program (grant ANR-09-BLAN-0009-01 - RUPXCUBE Project).

\section{References}

1. Suresh S (1998) Fatigue of materials, (2nd ed.) Cambridge University Press, Cambridge

2. Limodin N, Réthoré J, Buffière J-Y, Gravouil A, Hild F, Roux S (2009) Crack closure and stress intensity factor measurements in nodular graphite cast iron using three-dimensional correlation of laboratory x-ray microtomography images. Acta Mater 57(14):4090-4101

3. Weck A, Crawford THR, Borowiec A, Wilkinson DS, Preston JS (2007) Femtosecond laser-based fabrication of a new model material to study fracture. Appl Phys A Mater Sci Process 86:55-61

4. Buffiere J, Maire E, Adrien J, Masse J, Boller E (2010) In situ experiments with x ray tomography: an attractive tool for experimental mechanics. Exp Mech 50:289-305 
5. Limodin N, Réthoré J, Buffière J-Y, Hild F, Roux S, Ludwig W, Rannou J, Gravouil A (2010) Influence of closure on the 3d propagation of fatigue cracks in a nodular cast iron investigated by x-ray tomography and 3d volume correlation. Acta Mater 58(8):2957-2967

6. Roux S, Hild F, Viot P, Bernard D (2008) Three-dimensional image correlation from x-ray computed tomography of solid foam. Compos Part A Appl Sci Manuf 39(8):1253-1265

7. Réthoré J, Tinnes J-P, Roux S, Buffière J-Y, Hild F (2008) Extended three-dimensional digital image correlation (x3d-dic). C R Méc 336 (8):643-649

8. Williams ML (1957) On the stress distribution at the base of a stationary crack. ASME J Appl Mech 24:109-114 